\title{
A Simple and Safe Technique in Extracting Specimen after Sleeve Gastrectomy
}

\author{
Adem Yuksel ${ }^{1}$, Murat Coskun²
}

\begin{abstract}
Introduction:Today, minimally invasive surgery (laparoscopic, robotic) methods are becoming increasingly common. In the procedures in which the resection was performed with a minimally invasive surgical method, specimen removal can be time-consuming and complicated. In this study, we aimed to evaluate the results of laparoscopic sleeve gastrectomy specimens removed from a 12-mm trocar area without additional tools.

Materials and methods: Between January 2016 and December 2017, 129 patients underwent a laparoscopic sleeve gastrectomy for morbid obesity. In all patients, the specimen was removed from the abdomen from a 12-mm trocar area without additional tools.

Results: The mean specimen removal time was $2.38 \pm 1.9$ minutes. During the follow-up period, no wound infection and trocar hernia were observed in any patient.

Conclusion: The technique applied is minimally invasive, not time-consuming, and simple when compared to other techniques reported.

Keywords: Laparoscopic sleeve gastrectomy, Port hernia, Specimen extraction.

World Journal of Laparoscopic Surgery (2019): 10.5005/jp-journals-10033-1357
\end{abstract}

\section{INTRODUCTION}

Today, laparoscopic approach in gastrointestinal surgery is widely used owing to its advantages. In the procedures that the resection performed by the laparoscopic technique, different techniques such as expanding trocar incision, mini laparotomy from different regions, and removal from natural hole are used in the removal of the specimen from the abdomen., ${ }^{1,2}$ These different incisions and methods may occasionally lead to the elimination of some advantages (early postoperative recovery, decreased surgical site infection, esthetic appearance, decreased risk of hernia, etc.) of laparoscopic surgery. ${ }^{1}$

Many different procedures in surgical treatment of morbid obesity can be performed with the laparoscopic technique. Among these procedures, sleeve gastrectomy, in which the stomach is resected in the vertical axis, has been applied in increasing frequency in recent years. ${ }^{3}$ The removal of the specimen after sleeve gastrectomy can be time-consuming and complicated. There is no standard approach on this and many different techniques are applied.

In our study, it was aimed to evaluate the results of our patient group in which the sleeve gastrectomy specimen was removed from the 12-mm trocar area.

\section{Materials and Methods}

The study included patients who underwent laparoscopic sleeve gastrectomy for morbid obesity at Kocaeli Derince Training and Research Hospital between January 2016 and December 2017.

The decision was made as a result of the evaluation of the patients who were accepted according to the National Institutes of Health $(\mathrm{NIH})$ consensus criteria ${ }^{4}$ by the team consisting of surgery (gastrointestinal surgery, general surgery), endocrinology, psychiatry, gastroenterology, cardiology, chest diseases, sports medicine specialist, and dietitian team. A prophylaxis with $2 \mathrm{~g}$ of ceftriaxone was applied to all patients before the operation.
${ }^{1}$ Department of Gastrointestinal Surgery, Kocaeli Derince Training and Research Hospital, Kocaeli, Turkey

${ }^{2}$ Department of General Surgery, Kocaeli Derince Training and Research Hospital, Kocaeli, Turkey

Corresponding Author: Adem Yuksel, Department of Gastrointestinal Surgery, Kocaeli Derince Training and Research Hospital, Kocaeli, Turkey, Phone: +90 26231780 00, e-mail: ademyksel@yahoo.com

How to cite this article: Yuksel A, Coskun M. A Simple and Safe Technique in Extracting Specimen after Sleeve Gastrectomy. World J Lap Surg 2019;12(1):1-4.

Source of support: Nil

Conflict of interest: None

All operations were performed by the same surgical team consisting of two persons. Pneumoperitoneum was created in such a way to reach $12-14 \mathrm{~mm} \mathrm{Hg}$ by entering the abdomen with the help of a bladeless direct optical trocar (EndopathXcel; Ethicon EndoSurgery Inc., Cincinnati, Ohio) from $19 \mathrm{~cm}$ below the xiphoid and $4 \mathrm{~cm}$ lateral from the midline. The operation was performed with 3 pieces of $5 \mathrm{~mm}$ and 2 pieces of $12 \mathrm{~mm}$ trocars, one of which was the Nathanson retractor site (Fig. 1). All $12 \mathrm{~mm}$ trocars were bladeless optic trocar. Starting from approximately 2 to $6 \mathrm{~cm}$ proximal of pylorus, until the left diaphragmatic crura is revealed, the stomach was released by the large curvature with the help of LigaSure (Valleylab, Boulder, CO) or harmonic scalpel (EthiconEndosurgery, Cincinnati, $\mathrm{OH}$ ). The stomach was decompressed with a nasogastric or orogastric tube. The stomach was transected with the help of a $36 \mathrm{~F}$ bougie-guided endoscopic stapler (Ethicon Endosurgery, Cincinnati, OH). Transection was completed with 5 or 6 staplers. The gastrectomy-performed stomach was evaluated for leakage with 50-60 mL methylene blue. The resected stomach was held in the caudal end with a laparoscopic grasper and was taken $2-3 \mathrm{~cm}$ in a $12-\mathrm{mm}$ trocar parallel to the resection axis (Fig. 2). The specimen was removed from the abdomen with a trocar. The specimen was 


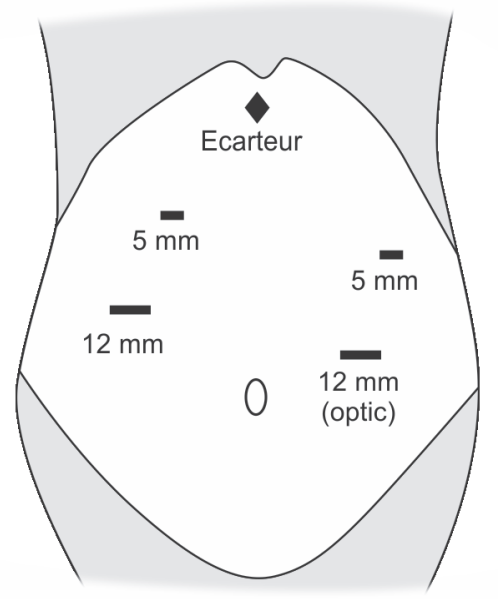

Fig. 1: Trocar locations

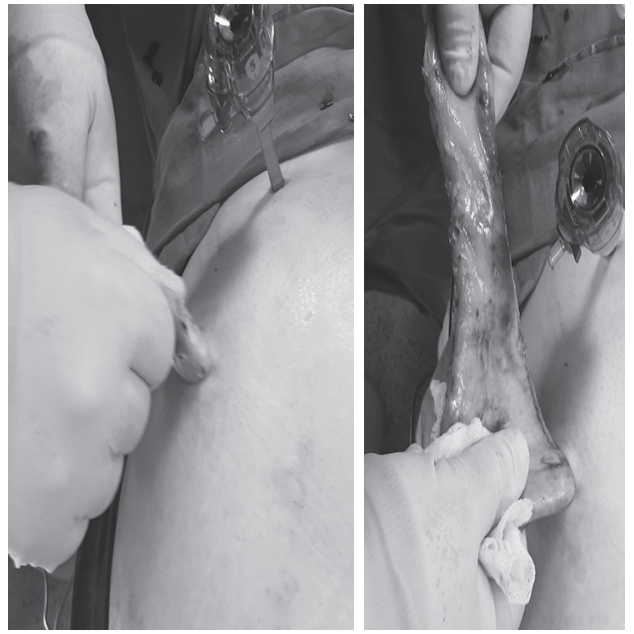

Fig. 3: Extraction of the entire specimen via the 12-mm trocar

extracted out of the abdomen by the large curvature, with the help of a gauze, by avoiding excessive traction (Fig. 3).

The patients' demographic characteristics (age, sex), body mass index (BMI), comorbid status, ASA score, intraoperative complications, specimen extraction time, and operation time were recorded. Specimen extraction time was obtained by the retrospective review of the operation video recordings in the first 55 cases, and from prospectively recorded data in subsequent cases. All patients were controlled on the 10th postoperative day, and on the 1st, 3rd, 6th, and 12th months by the surgical team. Wound-site infection status and trocar-site hernia status were recorded. Trocar site hernia status was evaluated by a clinical examination. In the statistical evaluation, a descriptive method was used.

\section{Results}

A total of 129 patients who underwent a laparoscopic sleeve gastrectomy were included in the study. Demographic characteristics (age, sex) and preoperative (body mass index, comorbidities, ASA score) characteristics of the patients are summarized in Table 1. All operations were completed laparoscopically. The sleeve gastrectomy was performed to one patient owing to gastric plication and to the other patient owing to the revision after vertical
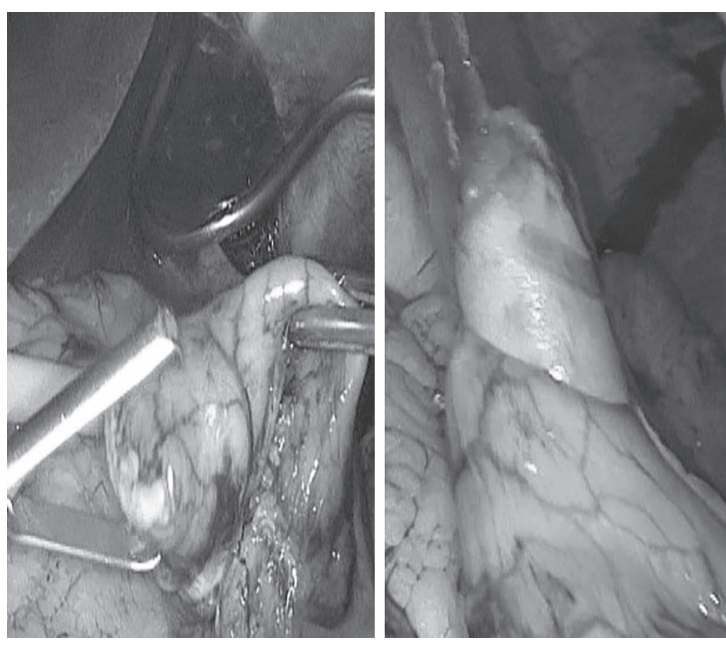

Fig. 2: Specimen grasping and pull into the $12-\mathrm{mm}$ trocar

Table 1: Patient characteristics

\begin{tabular}{lll}
\hline & & N: 129 \\
\hline Sex & Male & $24(18.6 \%)$ \\
Age & Female & $105(81.4 \%)$ \\
BMI & Mean \pm SD & $38 \pm 8.7$ \\
Comorbidity & Mean \pm SD & $45.7 \pm 6$ \\
& Hypertension (HT) & $28(21.7 \%)$ \\
& Diabetes mellitus (DM) & $51(39.5 \%)$ \\
ASA score & Pulmonary comorbidity & $16(12.4 \%)$ \\
& II & $98(76 \%)$ \\
& III & $31(24 \%)$ \\
\hline
\end{tabular}

Table 2: Intraoperative and postoperative results

\begin{tabular}{lll}
\hline & & $N: 129$ \\
\hline Operation & $\begin{array}{l}\text { Sleeve gastrectomy } \\
\text { Sleeve gastrectomy } \\
\text { + cholecystectomy }\end{array}$ & $117(90.7 \%)$ \\
Operation time (min) & Mean \pm SD & $75.7 \pm 19.4$ \\
$\begin{array}{l}\text { Specimen extraction time } \\
\text { (min) }\end{array}$ & Mean \pm SD & $2.38 \pm 1.19$ \\
$\begin{array}{l}\text { Specimen rupture } \\
\text { Wound infection }\end{array}$ & & $3(2.3 \%)$ \\
Port site hernia & & $0(0 \%)$ \\
\hline
\end{tabular}

band gastroplasty. A cholecystectomy was performed in 12 (9.3\%) patients in the same session. A cholecystectomy specimen was extracted from the same area. Four (3.1\%) patients had leakage after the sleeve gastrectomy. One of them was spontaneously closed. Others were treated with endoscopic treatment methods. The average operative time and specimen removal time were $75.7 \pm 19.4,2.38 \pm 1.9$ minutes, respectively. The fundus perforation was developed in three (2.3\%) patients during extraction. In addition to prophylactic antibiotherapy, antibiotic treatment was applied in the postoperative follow up of these patients. The average follow up period of the patients was $13.9 \pm 6.2$ months. During the follow up period, no wound site infection and trocar site hernia were seen in any patient (Table 2). 


\section{Discussion}

Nowadays, sleeve gastrectomy is used with increasing frequency in the surgical treatment of morbid obesity owing to some advantages such as preservation of normal anatomy and absorption capacity of small intestines, technically easy applicability and short operation time. $^{3}$ In this procedure, where successful results are obtained in the resolution of weight loss and comorbid conditions, 80-90\% of the stomach in vertical axis is resected. ${ }^{5}$ Removal of the specimen outside the abdomen after this wide resection can be a complicated, and prolonged duration of operation.

In laparoscopic surgery, there are questions looked for to be answered such as from which area the specimen should be removed, from how much width the specimen should be removed, how to reduce complications related to the area the specimen removed from, and whether the integrity of the specimen should be preserved in benign procedures. The answer to these questions was not standardized also in the sleeve gastrectomy procedure.

The method frequently applied in laparoscopic procedures is the extraction of the specimen by a mini laparotomy. In a study in which the specimen was removed by a mini-laparotomy after a sleeve gastrectomy, $5 \%$ wound site infection, 3.3\% hernia, and $8.3 \%$ hematoma in the site of extraction were detected. ${ }^{6}$ These results show that the specimen removal by mini-laparotomy reduce the advantages of the laparoscopic surgery.

Different techniques have been described, in which the specimen is removed after it was disintegrated. In one of these techniques, described by Mahmood et al., ${ }^{7}$ the specimen was disintegrated by a tissue disintegrator and removed from a $15-\mathrm{mm}$ trocar. Another technique is the technique by which Calin et al. ${ }^{8}$ extract the specimen from the $12 \mathrm{~mm}$ trocar site, by cutting and making the specimen thinner in the longitudinal axis intraabdominally. The main disadvantage of both techniques is the inability of histopathological examination of the specimen due to tissue disintegrity, and the risk of intraabdominal spread of gastric content. As a matter of fact, it was shown in several studies that in the sleeve gastrectomy specimen, there was a clinically significant histopathological finding at a rate of $3.3-5.8 \%$, and the histopathological examination of the specimen was necessary. ${ }^{9,10}$ In addition, it has been shown that intraabdominal spread of gastric content may cause a localized inflammatory response, adhesion, and intraabdominal abscess. ${ }^{11}$

The most commonly used technique for removal of specimen in sleeve gastrectomy is the removal of tissue from the trocar site by preserving tissue integrity. One of the controversial points in this technique is whether the wound site is protected with different auxiliary devices (wound site retractor, organ pouch) when removing the specimen. Studies have reported that the use of organ pouches or wound site retractors does not reduce infectious complications, prolonged operation time, and increased operation costs. ${ }^{12}$ In our technique, any auxiliary equipment was not used to protect the wound site and no wound site infection was seen in our series.

In order to reduce trocar-related complications after the sleeve gastrectomy, a technique involving a small number of patients with which the specimen was extracted transgastrically has been described. ${ }^{13}$ However, this technique requires laparoscopic and endoscopic experience, and the risk of intraabdominal spread of stomach contents is the most important disadvantage.

In a minimally invasive surgery, the overall goal is to use a smaller number of trocars with a smaller diameter. The use of larger trocar increases the risk of postoperative pain, patient comfort, and hernia risk in the trocar site. ${ }^{14}$ However, for the use of staplers in the sleeve gastrectomy, the minimum trocar diameter is $12 \mathrm{~mm}$, and there is only one study in the literature on removal of the specimen from a 12-mm trocar site by preserving the tissue integrity. In this study by Nassif et al., ${ }^{15}$ the fascia was extended with a Kocher clamp and the specimen was removed with the organ pouch. In our study, this technique has been modified. Without expanding the fascia and the usage of organ pouches, the specimen was extracted. Trocar-related complications such as wound site infection or trocar site hernia were not observed. According to the extraction time of the specimen, it was observed that the specimen was extracted in a time similar to those of the other studies. In three cases, it was observed that the specimen was ruptured from fundus, owing to insufficient decompression of the stomach and the rotation of the specimen on the reverse axis during extraction. Therefore, caution should be exercised to ensure that the stomach is sufficiently decompressed and not to cause any reverse rotation during traction.

\section{Conclusion}

According to the results of our study, the specimen can be safely extracted from the 12-mm trocar site after the sleeve gastrectomy, without the use of additional tools and without expansion of fascia and without prolonging the operation time.

\section{Ethical Committee Approval}

Ethical committee approval was obtained from University of Kocaeli (GOKAEK-2017/14.37 2017/300).

\section{References}

1. Lee $L$, Abou-Khalil M, et al. Incidence of incisional hernia in the specimen extraction site for laparoscopic colorectal surgery: systematic review and meta-analysis. Surg Endosc 2017 Dec;31(12):5083-5093. DOI: 10.1007/s00464-017-5573-2.

2. Leung AL, Cheung HY, et al. Advances in laparoscopic colorectal surgery: a review on NOTES and transanal extraction of specimen. Asian J Endosc Surg 2014 Jan;7(1):11-16. DOI: 10.1111/ases.12070.

3. Gagner M, Hutchinson C, et al. Fifth International Consensus Conference: current status of sleeve gastrectomy. Surg Obes Relat Dis 2016 May;12(4):750-756. DOI: 10.1016/j.soard.2016.01.022.

4. Gastrointestinal surgery for severe obesity: National Institutes of Health Consensus Development Conference Statement. Am J Clin Nutr 1992 Feb;55(2 Suppl):615S-619S. DOI: 10.1093/ajcn/55. 2.615s.

5. Cottam D, Qureshi FG, et al. Laparoscopic sleeve gastrectomy as an initial weight-loss procedure for high-risk patients with morbid obesity. Surg Endosc 2006;20(6):859-863. DOI: 10.1007/s00464-0050134-5.

6. Maietta P, Qureshi FG, et al. Retrieval of the gastric specimen following laparoscopic sleeve gastrectomy. Experience on 275 cases. Int J Surg 2016;28(Suppl 1):S124-S127. DOI: 10.1016/j.ijsu.2015.12.057.

7. Mahmood A, Silbergleit A. The utilization of a morcellator during laparoscopic sleeve gastrectomy. Technol Health Care 2006;14(6): 537-539.

8. Calin ML. Specimen extraction after laparoscopic sleeve gastrectomy. J Laparoendosc Adv Surg Tech A 2015 Apr;25(4):330. DOI: 10.1089/ lap.2014.0648.

9. Safaan T, Bashah M, et al. Histopathological Changes in Laparoscopic Sleeve Gastrectomy Specimens: Prevalence, Risk Factors, and Value of Routine Histopathologic Examination. Obes Surg 2017 Jul;27(7): 1741-1749. DOI: 10.1007/s11695-016-2525-1. 
10. Kopach $P, G e n e g a E M$, et al. The significance of histologic examination of gastrectomy specimens: a clinicopathologic study of 511 cases. Surg Obes Relat Dis 2017 Mar;13(3):463-467. DOI: 10.1016/ j.soard.2016.11.011.

11. Dotai T, Coker AM, et al. Transgastric large-organ extraction: the initial human experience. Surg Endosc 2013 Feb;27(2):394-399. DOI: 10.1007/s00464-012-2473-3.

12. Shoar S, Aboutaleb S, et al. Comparison of two specimen retrieval techniques in laparoscopic sleeve gastrectomy: what is the role of endobag? Surg Endosc 2017 Dec;31(12):4883-4887. DOI: 10.1007/ s00464-017-5434-z.
13. Gunkova P, Gunka I, et al. Laparoscopic sleeve gastrectomy for morbid obesity with natural orifice specimen extraction (NOSE). Bratisl Lek Listy 2015;116(7):422-425. DOI: 10.4149/BLL_2015_080.

14. Wang $Q$, Huang $L$, et al. Assessment of Port-Specific Pain After Gynecological Laparoscopy: A Prospective Cohort Clinical Trial. J Laparoendosc Adv Surg Tech A 2017 Jun;27(6):597-604. DOI: 10.1089/lap.2016.0340.

15. Bou Nassif G, Scetbun E, et al. Hand-Over-Hand Grasping Technique: A Fast and Safe Procedure for Specimen Extraction in Laparoscopic Sleeve Gastrectomy. Obes Surg 2017 May;27(5):1391. DOI: 10.1007/ s11695-017-2572-2. 